\title{
Novel VHL substrate targets SFMBT1 and ZHX2 may be important prognostic predictors in patients with ccRCC
}

\author{
YUFENG CHEN $^{1 *}$, LIANGSONG ZHU $^{2 *}$, SONG XUE $^{1}$, JIAN SHI $^{1}, \mathrm{CHUNFENG} \mathrm{HE}^{1}$ and QINGCHUAN ZHANG ${ }^{1}$ \\ ${ }^{1}$ Department of Urology, Putuo Hospital, Shanghai University of Traditional Chinese Medicine, Shanghai 200333; \\ ${ }^{2}$ Department of Urology, Zhongshan Hospital, Fudan University, Shanghai 200030, P.R. China
}

Received July 23, 2020; Accepted February 11, 2021

DOI: $10.3892 / \mathrm{ol} .2021 .12640$

\begin{abstract}
Renal cell carcinoma is one of the most malignant cancers, with limited prognostic prediction system. The present study aimed to determine the prognostic value of novel von Hippel-Lindau (VHL) substrate targets in predicting the outcome of clear cell renal cell carcinoma (ccRCC). A total of 97 patients with ccRCC were enrolled in the present study, and the tissue microarray that was constructed using 97 ccRCC samples was used for immunohistochemical analysis. Univariate and multivariate Cox regression analyses were performed to determine the independent prognostic factors. Reverse transcription-quantitative PCR analysis demonstrated that the mRNA expression levels of scm-like with four malignant brain tumor domains (SFMBT1) and zinc fingers and homeoboxes 2 (ZHX2) were upregulated in cancer tissues compared with adjacent normal tissues. Among the 97 patients with ccRCC, SFMBT1 expression was upregulated in $61.9 \%$ (60/97), while ZHX2 expression was upregulated in $52.6 \%$ (51/97). Overall survival (OS) and disease-free survival (DFS) analyses indicated that SFMBT1 or ZHX2 alone were of limited predictive value; however, the combined expression of these two targets (high SFMBT1 and high ZHX2 expression, SHZH group) was significantly associated with OS $(\mathrm{P}=0.0350)$ and DFS $(\mathrm{P}=0.0434)$. In addition, multivariate analysis identified $\mathrm{SHZH}$ as an independent prognostic factor in patients with ccRCC. Taken together, these results suggest that SFMBT1 and ZHX2 act as novel substrate targets of VHL and, to the best of our knowledge, the present study was
\end{abstract}

Correspondence to: Dr Qingchuan Zhang or Dr Chunfeng He, Department of Urology, Putuo Hospital, Shanghai University of Traditional Chinese Medicine, 164 Lanxi Road, Shanghai 200333, P.R. China

E-mail: qczhang888@yeah.net

E-mail: hechunfeng28@163.com

${ }^{*}$ Contributed equally

Key words: scm-like with four malignant brain tumor domains, zinc fingers and homeoboxes 2, prognostic value, clear cell renal cell carcinoma the first to provide insight on the co-expression of these two targets in representing a promising biomarker to predict the outcome of patients with ccRCC.

\section{Introduction}

Renal cell carcinoma ( $\mathrm{RCC}$ ) is the most lethal urological cancer, with an estimated 62,000 new cases diagnosed in the United States in 2020 (1). The most common histological subtype of RCC is clear cell (cc) RCC, which accounts for $\sim 80 \%$ of all cases (2). It is well-known that a germline mutation in the von Hippel-Lindau (VHL) gene plays an important role in ccRCC development, and the loss of function of the VHL protein (pVHL) may also be associated with resistance to cytotoxic chemotherapy $(3,4)$. Currently, renal surgery is extensively used for localized RCC, while the treatment options for patients with advanced or metastatic RCC (mRCC) are limited. Targeted therapy, such as sunitinib or sorafenib, is considered the standard first-line treatment for $\mathrm{mRCC}$, and is beneficial with regards to long-term survival (5). However, the development of drug resistance is considered inevitable following targeted therapy for 6-12 months (6). Currently, several prognostic prediction models have been used to assess the long-term outcomes of patients with RCC, such as the Mayo Clinic Stage, Size, Grade and Necrosis score (7) and the International Metastatic Renal Cell Carcinoma Database Consortium (8). In addition, several studies have reported that biomarker staining on postoperative pathology is an effective method for predicting prognosis (9-11). However, due to the heterogeneity of the molecular phenotype, single biomarker staining may have limited prediction power (12). Thus, a clinical outcome prediction model of multiple combined biomarkers with high accuracy is required for patients with RCC.

Previous studies have demonstrated that hypoxia-inducible factor (HIF)1a and HIF2a are regulated by pVHL-mediated ubiquitination. The loss of pVHL function may promote $\mathrm{HIFa}$ accumulation and translocation, thus contributing to the development of RCC $(13,14)$. Targeted therapy with tyrosine kinase inhibitors, which directly target the downstream factors of the $\mathrm{HIFa}$ pathway, is beneficial in the treatment of patients with RCC, while the HIF2a inhibitor exhibits limited efficiency (15). These findings suggest that there may be other signaling pathways involved in VHL-deficient RCC. Zhang et al (16) 
identified zinc fingers and homeoboxes 2 (ZHX2) as a novel VHL substrate factor that promotes the development of ccRCC. Subsequently, Zhu et al (17) reported that ZHX2 can directly target the MEK/ERK signaling pathway in ccRCC cell lines and induce sunitinib resistance by overexpressing ZHX2. In addition, scm-like with four malignant brain tumor domains (SFMBT1) was also identified as a candidate VHL target by genome-wide screening and SFMBT1 is considered an oncogenic driver in ccRCC (18), however, its prognostic value remains unclear.

The predictive ability of combining multiple novel VHL substrate targets is yet to be investigated. Thus, the present study aimed to investigate the clinical significance and prognostic value of SFMBT1 combined with ZHX2, in the hope to better understand the role of these novel VHL substrates in targeted therapeutic intervention.

\section{Materials and methods}

Patients and tissues. A total of 97 patients, including 68 (70.1\%) men and 29 (29.9\%) women were enrolled in the present study. with confirmed ccRCC pathology, who underwent radical nephrectomy at Putuo hospital between January 2010 and December 2015, were enrolled in the present study. Follow-up examinations (from January 2010 to January 2020, including blood tests and CT/MRI, were performed every 6 months in the first 3 years, and once a year after that. All medical records and laboratory information were collected, including age, surgery time, sex, tumor size and tumor stage. Those who received chemoradiotherapy prior to selection, and had incomplete clinical records or missed follow-up were excluded from the present study.

A total of five paired fresh tumor tissues and adjacent normal tissues were collected ( $>3 \mathrm{~cm}$ apart) for RNA extraction and to detect mRNA expression. The present study was approved by the Ethical Review Boards of Putuo Hospital (Shanghai, China, approval no. 20200130) and written informed consent was provided by all patients prior to the study start.

Database analysis and survival data. The expression levels of SFMBT1 and ZHX2 in kidney cancer were assessed using the Gene Expression Profiling Interactive Analysis (GEPIA) database (http://gepia.cancer-pku.cn). In addition, the GEPIA database was used to retrieve information on the prognostic values of SFMBT1 and ZHX2 in kidney cancer, including overall survival (OS) and disease-free survival (DFS). The survival rates were measured according to the data from 864 patients.

Tissue microarrays and immunohistochemistry (IHC). The tissue microarray was constructed based on the tumor samples of the 97 patients with ccRCC. The protocol was as follows: 1) Tissue chip was soaked in xylene for $10 \mathrm{~min}$ (three times); 2) hydrated with $100 \%$ ethanol (three times), $95 \%$ ethanol (once), $85 \%$ ethanol (once), 75\% ethanol (once) and washed with distilled water (three times); 3) washed with PBS (5 min each time); 4) incubated with $3 \% \mathrm{H}_{2} \mathrm{O}_{2}$ for 20 min at room temperature to inhibit endogenous peroxidase activity and washed with PBS (three times); 5) antigen repair: Incubated with $1 \mathrm{mmol} / 1$ EDTA antigen repair solution for $10 \mathrm{~min}$;
6) Incubated with $10 \%$ goat serum at room temperature for $10 \mathrm{~min} ; 7)$ incubated with primary antibodies against SFMBT1 (1:200 dilution; cat. no. A303-221A; Bethyl Laboratories, Inc.) and ZHX2 (1:50 dilution; cat. no. GTX112232; GeneTex Inc.) overnight at $4^{\circ} \mathrm{C}$, and washed with PBS (three times); 8) incubated with secondary antibody at room temperature for $1 \mathrm{~h}$, washed with PBS (three times). DAB chromogen was added to observe the chromogen state under the light microscope (magnification, $x 40$ ), rinsed with distilled water. 10) Stained with hematoxylin at room temperature: Soak the tissue chip in hematoxylin solution (prepared at 1:20); 11) dehydrated with $75 \%$ ethanol for $10 \mathrm{sec}, 85 \%$ ethanol for $10 \mathrm{sec}, 95 \%$ ethanol for $10 \mathrm{sec}, 100 \%$ ethanol for $5 \mathrm{~min}$ (three times) and xylene for $5 \mathrm{~min}$ (three times); 12) Sequestration. IHC analysis was performed as previously described (19). IHC analysis was performed to detect the expression levels of the novel VHL substrate targets, SFMBT1 and ZHX2. The location and expression of the targets were independently confirmed by two pathologists at Putuo hospital.

The IHC staining score was the sum of the staining percentage and the intensity degree. Staining percentage was calculated as follows: $1,0-25 ; 2,26-50 ; 3,51-75$ and $4,>75 \%$, while intensity degree was calculated as follows: 0 , negative; 1 , weak; 2 , moderate and 3 , strong. An IHC score $<6$ was classified as the low expression group, while an IHC score $\geq 6$ was classified as the high expression group (12). All patients were divided into two groups, according to the combined expression levels of SFMBT1 and ZHX2. Patients with high SFMBT1 and ZHX2 expression levels were classified into the SHZH group.

Reverse transcription-quantitative (RT-q)PCR. Total RNA was extracted from fresh tissue samples using TRIzol ${ }^{\circledR}$ reagent (Thermo Fisher Scientific, Inc., cat. no. 15596018) and trichloromethane (Nanjing KeyGen Biotech Co., Ltd., cat. no. C07615202). Total RNA was reverse transcribed into cDNA using the HiScript II Q RT SuperMix RT kit (+gDNA wiper, cat. no. R223-01; Vazyme Biotech Co., Ltd.) at $50^{\circ} \mathrm{C}$ for $15 \mathrm{~min}$ and $85^{\circ} \mathrm{C}$ for $5 \mathrm{sec}$. qPCR was subsequently performed using the SYBR Green kit (Takara Biotechnology Co., Ltd.), according to the manufacturer's protocol. The following thermocycling conditions were used for qPCR: $95^{\circ} \mathrm{C}$ for $30 \mathrm{sec}$, cyclic reaction with $95^{\circ} \mathrm{C}$ for $10 \mathrm{sec}, 60^{\circ} \mathrm{C}$ for $20 \mathrm{sec}$ and $72^{\circ} \mathrm{C}$ for $20 \mathrm{sec}$ in 40 rounds. The following primer sequences were used for qPCR: SFMBT1 forward, 5'-TGCCACCATTTG CTGAT-3' and reverse, 5'-TTTGTCCACCTCCATTCTG-3; ZHX2 forward, 5'-CTGCCTTAGCCCCACAC-3' and reverse, 5'-TGCTACCCAGTTCTCCCA-3'; and GAPDH forward, 5'-ACAGTCAGCCGCATCTTCTT-3' and reverse, 5'-GAC AAGCTTCCCGTTCTCAG-3'. Relative expression levels were calculated using the $2^{-\Delta \Delta C a}$ method (20) normalized to the internal reference gene GAPDH.

Statistical analysis. Statistical analysis was performed using SPSS 19.0 software (IBM Corp.) and GraphPad Prism software (version 6; GraphPad Software, Inc.). Paired Student's t-test or the Mann-Whitney test were used to compare continuous variables, while Pearson's $\chi^{2}$ or Fisher's exact tests were used to compare categorical variables. OS and DFS analyses were performed using the Kaplan-Meier 
A

SFMBT1

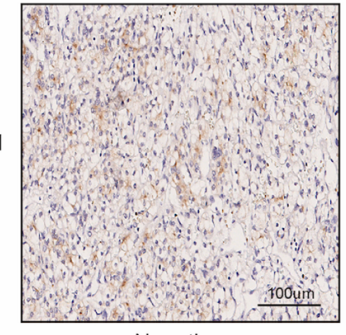

Negative

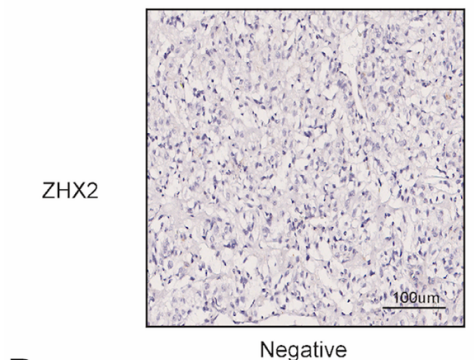

B
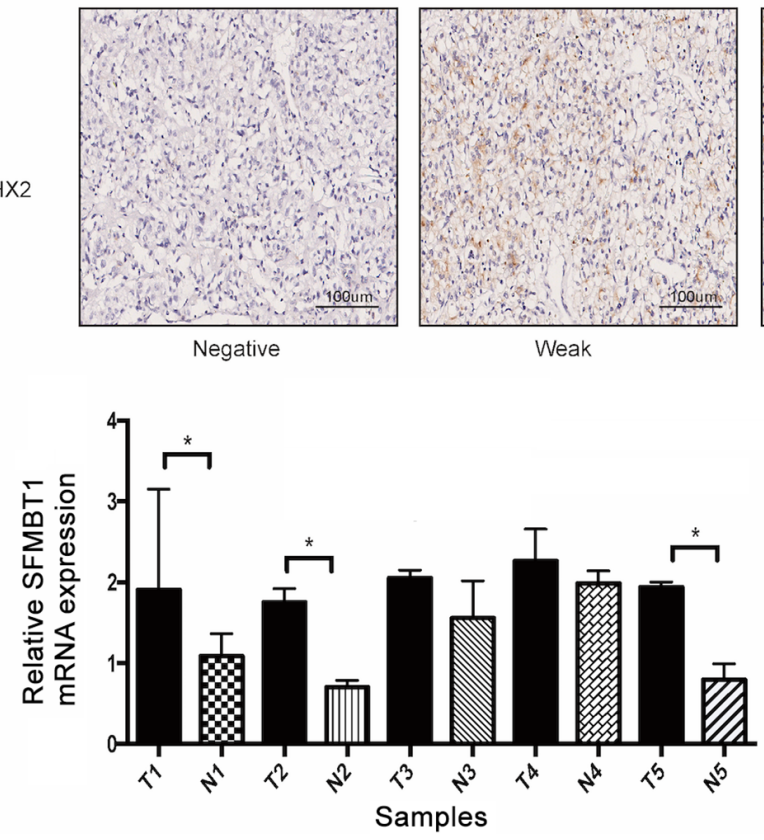

Weak

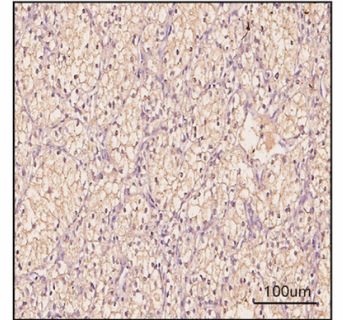

Moderate

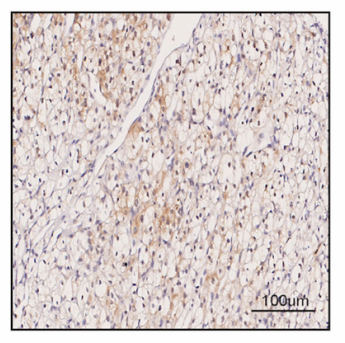

C

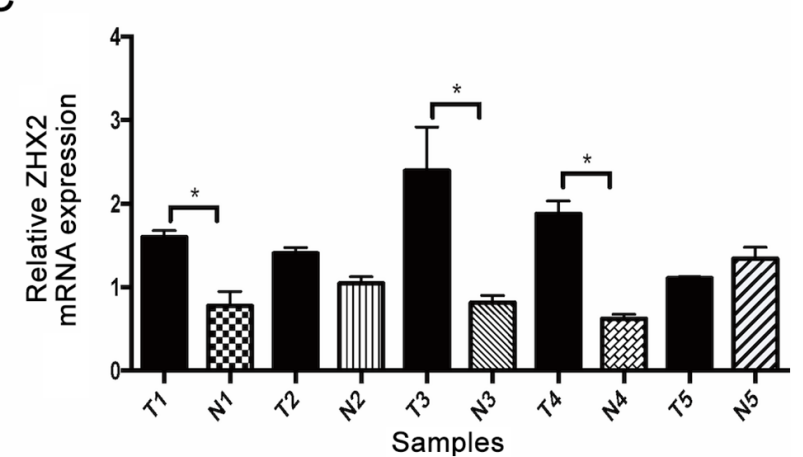

Figure 1. Expression levels of SFMBT1 and ZHX2 in ccRCC samples. (A) Representative images of SFMBT1 and ZHX2 expression levels in ccRCC samples. Scale bar, $100 \mu \mathrm{m}$. (B) Relative mRNA expression levels of (B) SFMBT1 and (C) ZHX2 in fresh ccRCC tissues and adjacent normal tissues. "P<0.05. SFMBT1, scm-like with four malignant brain tumor domains; ZHX2, zinc fingers and homeoboxes 2; ccRCC, clear cell renal cell carcinoma; T, tumor; N, normal.

method and log-rank test. Univariate Cox regression analysis was performed to determine the prognostic factors, and significant variables were further analyzed via multivariate Cox regression analysis to determine the independent prognostic factors. $\mathrm{P}<0.05$ was considered to indicate a statistically significant difference.

\section{Results}

SFMBT1 and ZHX2 expression in ccRCC. The GEPIA database was searched, which indicated that SFMBT1 expression was downregulated in ccRCC tissues, while ZHX2 expression was relatively upregulated in ccRCC tissue (Fig. S1). IHC analysis was performed to detect the expression levels of SFMBT1 and ZHX2 in ccRCC samples. As presented in Fig. 1A, SFMBT1 was localized in the nucleus and cytoplasm, while ZHX2 was predominantly localized in the nucleus, and both proteins were upregulated in tumor tissues compared with adjacent normal tissues. RT-qPCR analysis was subsequently performed to confirm these results, which demonstrated that the mRNA expression levels of SFMBT1 and ZHX2 were upregulated in tumor tissues compared with adjacent normal tissues (Fig. 1B and C).

The detailed clinicopathological characteristics of all patients are presented in Table I. A total of 68 (70.1\%) men and
$29(29.9 \%)$ women were enrolled in the present study. SFMBT1 expression was upregulated in 61.9\% (60/97) of patients with ccRCC, while ZHX2 expression was upregulated in 52.6\% (51/97) of patients. Notably, high SFMBT1 expression was significantly associated with advanced tumor status (TNM stage (21) and Fuhrman grade (22), while high ZHX2 expression was significantly associated with advanced Fuhrman grade. Taken together, these results suggest that SFMBT1 and ZHX2 act as oncogenes in ccRCC.

Prognostic values of SFMBT1 and ZHX2 in patients with $c c R C C$. To determine the prognostic values of SFMBT1 and ZHX2 in ccRCC, patients were divided into two groups, according to the combined expression levels of SFMBT1 and ZHX2. Of the 97 patients, 32 patients were classified into the high expression group (SHZH group), while 29 patients were classified into the low expression group (SLZL group); the remaining 36 patients were defined as other group (SLZH or SHZL groups).

Kaplan-Meier survival analysis was performed to determine the prognostic values of SFMBT1 and ZHX2. As presented in Fig. 2, the 5-year survival rates were 78.3 and $89.2 \%$ in patients with high and low SFMBT1 expression, respectively. In addition, patients with high SFMBT1 expression had a significantly lower OS rate $(\mathrm{P}=0.0179)$ than 
Table I. Clinicopathological characteristics of patients with clear cell renal cell carcinoma $(\mathrm{n}=97)$.

\begin{tabular}{|c|c|c|c|c|c|c|c|}
\hline \multirow[b]{2}{*}{ Characteristic } & \multirow[b]{2}{*}{ Patients, n (\%) } & \multicolumn{3}{|c|}{ Tumoral SFMBT1 expression } & \multicolumn{3}{|c|}{ Tumoral ZHX2 expression } \\
\hline & & Low & High & $\mathrm{P}$-value & Low & High & P-value \\
\hline Patients & $97(100.0)$ & 37 & 60 & & 46 & 51 & \\
\hline Sex & & & & 0.582 & & & 0.544 \\
\hline Male & $68(70.1)$ & 26 & 42 & & 32 & 36 & \\
\hline Female & $29(29.9)$ & 11 & 18 & & 14 & 15 & \\
\hline Age, years & & & & 0.507 & & & 0.531 \\
\hline$\leq 55$ & $46(47.4)$ & 18 & 28 & & 24 & 22 & \\
\hline$>55$ & $51(52.6)$ & 19 & 32 & & 22 & 29 & \\
\hline TNM stage & & & & 0.035 & & & 0.237 \\
\hline $\mathrm{I} / \mathrm{II}$ & $87(92.8)$ & 36 & 51 & & 43 & 44 & \\
\hline III/IV & $10(7.2)$ & 1 & 9 & & 3 & 7 & \\
\hline Fuhrman grade & & & & 0.046 & & & 0.043 \\
\hline $\mathrm{I} / \mathrm{II}$ & 76 (78.4) & 33 & 43 & & 40 & 36 & \\
\hline III/IV & $21(21.6)$ & 4 & 17 & & 6 & 15 & \\
\hline Tumor size, $\mathrm{cm}$ & & & & 0.298 & & & 0.413 \\
\hline$\leq 4$ & $40(41.2)$ & 17 & 23 & & 20 & 20 & \\
\hline$>4$ & $57(58.8)$ & 20 & 37 & & 26 & 31 & \\
\hline
\end{tabular}

TNM, tumor-node-metastasis; SFMBT1, scm-like with four malignant brain tumor domains; ZHX2, zinc fingers and homeoboxes 2.
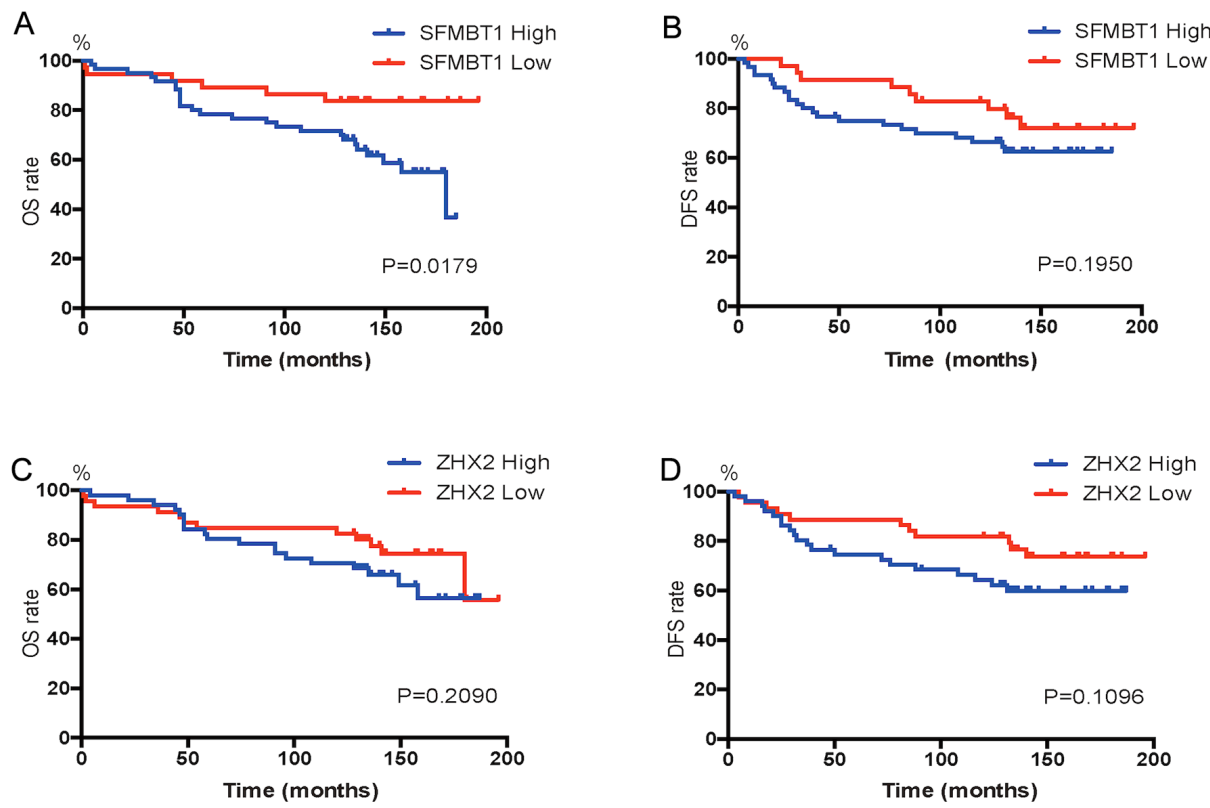

Figure 2. Kaplan-Meier survival analysis of patients with clear cell renal cell carcinoma, based on SFMBT1 and ZHX2 expression levels. (A) OS rate and (B) DFS rate curves based on SFMBT1 expression. (C) OS rate and (D) DFS rate curves based ZHX2 expression. SFMBT1, scm-like with four malignant brain tumor domains; ZHX2, zinc fingers and homeoboxes 2; OS, overall survival; DFS, disease-free survival.

those with low SFMBT1 expression. DFS was comparable between patients with high and low SFMBT1 expression levels. Notably, no significant differences were observed in the OS and DFS rates between patients with high and low ZHX2 expression.

The GEPIA database was searched to retrieve information on the prognostic values of SFMBT1 and ZHX2. As presented in Fig. S2, no significant differences in OS and DFS analyses were observed. Subsequently, the prognostic value of SFMBT1 combined with ZHX2 in ccRCC was assessed. The OS and DFS rates in different groups are presented in Fig. 3A and B, there was no significant difference between these groups, and patients in the SHZH group had a worst outcome. As presented in Fig. 3C and D, patients in the SHZH group had significantly 
Table II. Univariate and multivariate Cox regression analyses based on overall survival.

\begin{tabular}{|c|c|c|c|c|c|c|}
\hline \multirow[b]{2}{*}{ Variable } & \multicolumn{3}{|c|}{ Univariate analysis } & \multicolumn{3}{|c|}{ Multivariate analysis } \\
\hline & HR & $95 \% \mathrm{CI}$ & P-value & HR & $95 \% \mathrm{CI}$ & P-value \\
\hline SFMBT1 expression (Low vs. High) & 0.583 & $0.435-1.491$ & 0.042 & 0.582 & $0.234-1.283$ & 0.152 \\
\hline ZHX2 expression (Low vs. High) & 0.382 & $0.273-0.825$ & 0.083 & - & - & - \\
\hline SHZH expression (SHZH vs. Other) & 0.343 & $0.213-0.628$ & $0.003^{\mathrm{b}}$ & 0.252 & $0.203-0.755$ & $0.021^{\mathrm{a}}$ \\
\hline Age, years ( $\leq 55$ vs. $>55)$ & 1.342 & $0.893-1.582$ & $0.039^{\mathrm{a}}$ & 1.120 & $0.723-1.321$ & 0.342 \\
\hline TNM stage (I/II vs. III/IV) & 5.823 & $2.783-10.809$ & $<0.001^{\mathrm{c}}$ & 3.012 & $2.783-8.283$ & $0.031^{\mathrm{a}}$ \\
\hline Fuhrman grade (I/II vs. III/IV) & 6.261 & $3.172-9.632$ & $0.001^{\mathrm{b}}$ & 4.391 & $1.653-7.402$ & $0.093^{\mathrm{a}}$ \\
\hline Tumor size, $\mathrm{cm}(\leq 4 \mathrm{vs.}>4)$ & 3.869 & $1.873-8.846$ & $0.021^{\mathrm{a}}$ & 2.097 & $1.072-7.842$ & $0.048^{\mathrm{a}}$ \\
\hline
\end{tabular}

${ }^{\mathrm{a}} \mathrm{P}<0.05 ;{ }^{\mathrm{b}} \mathrm{P}<0.01 ;{ }^{\mathrm{c}} \mathrm{P}<0.001$. SFMBT1, scm-like with four malignant brain tumor domains; ZHX2, zinc fingers and homeoboxes 2 ; SHZH, SFMBT1 high and ZHX2 high; TNM, tumor-node-metastasis; HR, hazard ratio; CI, confidence interval; -, not applicable.

Table III. Univariate and multivariate Cox regression analyses based on disease-free survival.

\begin{tabular}{|c|c|c|c|c|c|c|}
\hline \multirow[b]{2}{*}{ Variable } & \multicolumn{3}{|c|}{ Univariate analysis } & \multicolumn{3}{|c|}{ Multivariate analysis } \\
\hline & HR & $95 \% \mathrm{CI}$ & P-value & HR & $95 \% \mathrm{CI}$ & P-value \\
\hline SFMBT1 expression (Low vs. High) & 0.472 & $0.235-1.145$ & 0.148 & - & - & - \\
\hline ZHX2 expression (Low vs. High) & 0.455 & $0.334-0.893$ & 0.273 & - & - & - \\
\hline SHZH expression (SHZH vs. Other) & 0.388 & $0.234-0.962$ & $0.009^{\mathrm{b}}$ & 0.394 & $0.303-1.274$ & $0.016^{\mathrm{a}}$ \\
\hline Age, years ( $\leq 55$ vs. $>55)$ & 1.538 & $0.712-1.863$ & $0.041^{\mathrm{a}}$ & 1.684 & $0.592-1.702$ & 0.281 \\
\hline TNM stage (I/II vs. III/IV) & 4.172 & $2.653-8.082$ & $<0.001^{\mathrm{c}}$ & 3.712 & $2.301-9.729$ & $0.030^{\mathrm{a}}$ \\
\hline Fuhrman grade (I/II vs. III/IV) & 6.261 & $3.172-9.632$ & $0.001^{\mathrm{b}}$ & 4.391 & $1.601-8.784$ & 0.086 \\
\hline Tumor size, $\mathrm{cm}(\leq 4$ vs. $>4)$ & 3.142 & $1.421-6.429$ & $0.039^{\mathrm{a}}$ & 2.537 & $1.694-8.893$ & 0.072 \\
\hline
\end{tabular}

${ }^{\mathrm{a}} \mathrm{P}<0.05 ;{ }^{\mathrm{b}} \mathrm{P}<0.01 ;{ }^{\mathrm{C}} \mathrm{P}<0.001$. SFMBT1, scm-like with four malignant brain tumor domains; ZHX2, zinc fingers and homeoboxes 2; SHZH, SFMBT1 high and ZHX2 high; TNM, tumor-node-metastasis; HR, hazard ratio; CI, confidence interval; -, not applicable.

A
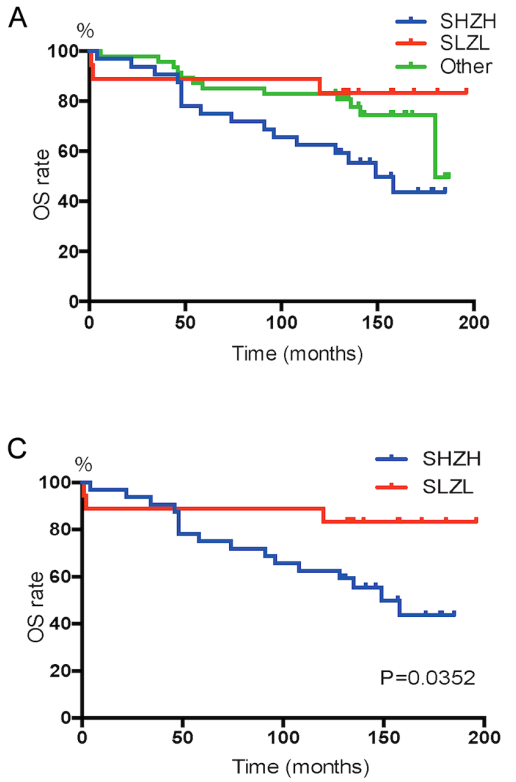

B

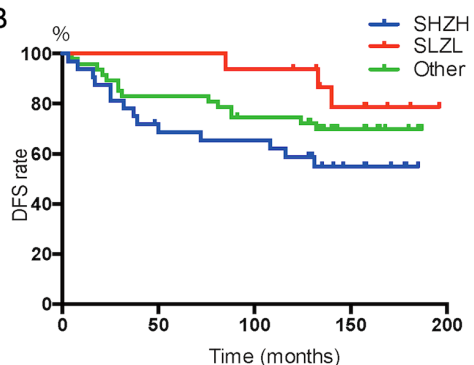

D

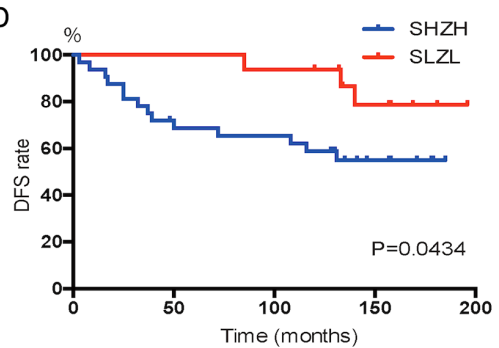

Figure 3. Kaplan-Meier survival analysis of patients with clear cell renal cell carcinoma, based on combined expression levels of SFMBT1 and ZHX2. (A) OS rate and (B) DFS rate curves based on combined expression levels of SFMBT1 and ZHX2. (C) OS rate and (D) DFS rate analyses of patients in the SHZH and SLZL groups. SFMBT1, scm-like with four malignant brain tumor domains; ZHX2, zinc fingers and homeoboxes 2; OS, overall survival; DFS, disease-free survival; SHZH, SFMBT1 high and ZHX2 high; SLZL, SFMBT1 low and ZHX2 low. 
worse OS $(\mathrm{P}=0.0352)$ and DFS $(\mathrm{P}=0.0434)$ rates compared with patients in the SLZL group.

Combined expression of SFMBT1 and ZHX2 may be an independent factor in ccRCC prognosis. The prognostic value of different factors on OS and DFS was determined via univariate and multivariate Cox regression analyses (Tables II and III). Univariate analysis demonstrated that SFMBT1 expression was significantly associated with OS rate [hazard ratio (HR), 0.583; $\mathrm{P}=0.042$; Table II], while $\mathrm{SHZH}$ (combined expression group), age, TNM stage, Fuhrman grade and tumor size were significantly associated with OS and DFS rates (Tables II and III). Multivariate analysis demonstrated that $\mathrm{SHZH}$ was an independent predictive factor of OS (HR, 0.252; $\mathrm{P}=0.021)$ and $\mathrm{DFS}(\mathrm{HR}, 0.394 ; \mathrm{P}=0.016)$ rates for patients with ccRCC. Collectively, these results suggest that the combined expression of SFMBT1 and ZHX2 may be used as a promising prognostic factor in predicting the outcomes of patients with ccRCC.

\section{Discussion}

The present study investigated the expression levels of SFMBT1 and ZHX2 in ccRCC tissues. The results demonstrated that both genes were relatively upregulated in cancer tissues compared with adjacent normal tissues. As both genes are considered VHL substrate factors, their combined prediction value in ccRCC prognosis was assessed in the present study. The association between SFMBT1/ZHX2 expression and clinicopathological characteristics demonstrated that both genes act as oncogenes in ccRCC development. Furthermore, Kaplan-Meier survival analysis indicated that the combined expression of SFMBT1 and ZHX2 was significantly associated with OS and DFS, and patients in the SHZH group had lower survival rates. Thus, $\mathrm{SHZH}$ was identified as an independent predictor for ccRCC outcomes.

SFMBT1 is a member of the MBT domain-containing protein family, which plays a critical role in chromatin regulation (23). Tang et al (24) reported that SFMBT1 is an essential part of LSD1 in genetic modification, and SFMBT1 is associated with epithelial-to-mesenchymal transition and poor prognosis in human breast cancer. Furthermore, Liu et al (18) demonstrated that SFMBT1 is regulated by pVHL via a prolyl hydroxylation and proteasomal degradation process, similar to HIFs and ZHX2. Their research found that overexpression of SFMBT1 promotes cell proliferation and tumor growth in ccRCC. Furthermore, the importance of the pVHL-SFMBT1-SPHK1 signaling pathway in ccRCC development was identified.

The role of ZHX2 in cancer remains controversial. Previous studies have reported that ZHX2 plays a dual role as both an oncogene and tumor suppressor (25-27). Zhang et al (16) identified ZHX2 as a VHL novel substrate factor, and ZHX2 is regulated by pVHL-mediated degradation. The in vitro experiments demonstrated that $\mathrm{ZHX} 2$ depletion can significantly downregulate NF- $\kappa \mathrm{B}$ activation, thereby inhibiting the proliferation and tumor-forming ability of ccRCC cells. Zhu et al (17) used a ZHX2 overexpression lentivirus to transfect $786-\mathrm{O}$ and CAKI-1 cells for lineage reprogramming, and the transcriptome analysis revealed that ZHX2 overexpression can directly activate the MEK/ERK1/2 signaling pathway, which in turn activates ccRCC angiogenesis and development.

The two novel VHL substrate factors, SFMBT1 and ZHX2, appear to have the same mechanism of action; however, they may activate different signaling pathways in ccRCC cell lines. Further studies are required to confirm the prognostic value of combining the transcription factors in RCC. To the best of our knowledge, the present study was the first to investigate the co-expression level of these two genes in ccRCC samples and assess their association with prognostic outcomes. In the present study, survival analysis demonstrated that combined high expression levels of SFBMT1 and ZHX2 were associated with poor clinical outcomes in patients with ccRCC. However, larger sample sizes and multicenter data are required to verify the results presented here.

In conclusion, SHZH appears to be a promising prognostic predictor in patients with ccRCC, and the result of the present study provide novel insight into advanced ccRCC treatment and follow-up.

\section{Acknowledgements}

Not applicable.

\section{Funding}

No funding was received.

\section{Availability of data and materials}

The datasets used and/or analyzed during the present study are available from the corresponding author upon reasonable request.

\section{Authors' contributions}

YFC and LSZ drafted the initial manuscript. YFC and LSZ conceived the present study and analyzed and interpreted the data. YFC, LSZ, SX and JS collected the data and prepared the figures and tables. CFH and QCZ performed the experiments and designed and developed the database. YFC and QCZ confirmed the authenticity of all the raw data. QCZ critically revised the manuscript for important intellectual content. All authors have read and approved the final manuscript.

\section{Ethics approval and consent to participate}

The present study was approved by the Ethical Review Boards of Putuo Hospital (Shanghai, China; approval no. 20200130), and written informed consent was provided by all patients prior to the study start.

\section{Patient consent for publication}

Not applicable.

\section{Competing interests}

The authors declare that they have no competing interests. 


\section{References}

1. Siegel RL, Miller KD and Jemal A: Cancer statistics, 2020. CA Cancer J Clin 70: 7-30, 2020.

2. Escudier B, Porta C, Schmidinger M, Rioux-Leclercq N, Bex A, Khoo V, Grünwald V, Gillessen S and Horwich A; ESMO Guidelines Committee. Electronic address: clinicalguidelines@ esmo.org: Renal cell carcinoma: ESMO Clinical Practice Guidelines for diagnosis, treatment and follow-up. Ann Oncol 30: 706-720, 2019.

3. Cancer Genome Atlas Research Network: Comprehensive molecular characterization of clear cell renal cell carcinoma. Nature 499: 43-49, 2013.

4. Kaelin WG Jr: Molecular basis of the VHL hereditary cancer syndrome. Nat Rev Cancer 2: 673-682, 2002.

5. Escudier B, Szczylik C, Porta C and Gore M: Treatment selection in metastatic renal cell carcinoma: Expert consensus. Nat Rev Clin Oncol 9: 327-337, 2012.

6. Harshman LC, Xie W, Bjarnason GA, Knox JJ, MacKenzie M, Wood L, Srinivas S, Vaishampayan UN, Tan MH, Rha SY, et al: Conditional survival of patients with metastatic renal-cell carcinoma treated with VEGF-targeted therapy: A population-based study. Lancet Oncol 13: 927-935, 2012.

7. Ficarra V, Novara G, Galfano A, Brunelli M, Cavalleri S, Martignoni G and Artibani W: The 'stage, size, grade and necrosis' score is more accurate than the University of California los angeles integrated staging system for predicting cancer-specific survival in patients with clear cell renal cell carcinoma. BJU Int 103: 165-170, 2009.

8. Ko JJ, Xie W, Kroeger N, Lee JL, Rini BI, Knox JJ, Bjarnason GA, Srinivas S, Pal SK, Yuasa T, et al: The international metastatic renal cell Carcinoma database consortium model as a prognostic tool in patients with metastatic renal cell carcinoma previously treated with first-line targeted therapy: A population-based study. Lancet Oncol 16: 293-300, 2015.

9. Zhu L, Wang J, Kong W, Huang J, Dong B, Huang Y, Xue W and Zhang J: LSD1 inhibition suppresses the growth of clear cell renal cell carcinoma via upregulating P21 signaling. Acta Pharm Sin B 9: 324-334, 2019.

10. Liao Y, Xiao H, Cheng M and Fan X: Bioinformatics analysis reveals biomarkers with cancer stem cell characteristics in lung squamous cell carcinoma. Front Genet 11: 427, 2020.

11. Liao Y, Wang Y, Cheng M, Huang C and Fan X: Weighted gene coexpression network analysis of features that control cancer stem cells reveals prognostic biomarkers in lung adenocarcinoma. Front Genet 11: 311, 2020.

12. Zhu L, Ding R, Zhang J, Zhang J and Lin Z: Cyclin-dependent kinase 5 acts as a promising biomarker in clear cell renal cell carcinoma. BMC Cancer 19: 698, 2019.

13. Ivan M, Kondo K, Yang H, Kim W, Valiando J, Ohh M, Salic A, Asara JM, Lane WS and Kaelin WG Jr: HIFalpha targeted for VHL-mediated destruction by proline hydroxylation: Implications for O2 sensing. Science 292: 464-468, 2001.

14. Hon WC, Wilson MI, Harlos K, Claridge TD, Schofield CJ, Pugh CW, Maxwell PH, Ratcliffe PJ, Stuart DI and Jones EY: Structural basis for the recognition of hydroxyproline in HIF-1 alpha by pVHL. Nature 417: 975-978, 2002.
15. Cho H, Du X, Rizzi JP, Liberzon E, Chakraborty AA, Gao W, Carvo I, Signoretti S, Bruick RK, Josey JA, et al: On-target efficacy of a HIF-2 $\alpha$ antagonist in preclinical kidney cancer models. Nature 539: 107-111, 2016.

16. Zhang J, Wu T, Simon J, Takada M, Saito R, Fan C, Liu XD, Jonasch E, Xie L, Chen X, et al: VHL substrate transcription factor ZHX2 as an oncogenic driver in clear cell renal cell carcinoma. Science 361: 290-295, 2018.

17. Zhu L, Ding R, Yan H, Zhang J and Lin Z: ZHX2 drives cell growth and migration via activating MEK/ERK signal and induces sunitinib resistance by regulating the autophagy in clear cell renal cell carcinoma. Cell Death Dis 11: 337, 2020.

18. Liu X, Simon JM, Xie H, Hu L, Wang J, Zurlo G, Fan C, Ptacek TS, Herring L, Tan X, et al: Genome-wide screening identifies SFMBT1 as an oncogenic driver in cancer with VHL loss. Mol Cell 77: 1294-1306.e5, 2020.

19. Matos LL, Trufelli DC, de Matos MG and da Silva Pinhal MA: Immunohistochemistry as an important tool in biomarkers detection and clinical practice. Biomark Insights 5: 9-20, 2010.

20. Livak KJ and Schmittgen TD: Analysis of relative gene expression data using real-time quantitative PCR and the 2(-Delta Delta C(T)) method. Methods 25: 402-408, 2001.

21. Edge SB and Compton CC: The American joint committee on cancer: The 7th edition of the AJCC cancer staging manual and the future of TNM. Ann Surg Oncol 17: 1471-1474, 2010.

22. Fuhrman SA, Lasky LC and Limas C: Prognostic significance of morphologic parameters in renal cell carcinoma. Am J Surg Pathol 6: 655-663, 1982

23. Bonasio R, Lecona E and Reinberg D: MBT domain proteins in development and disease. Semin Cell Dev Biol 21: 221-230, 2010.

24. Tang M, Shen H, Jin Y, Lin T, Cai Q, Pinard MA, Biswas S, Tran Q, Li G, Shenoy AK, et al: The malignant brain tumor (MBT) domain protein SFMBT1 is an integral histone reader subunit of the LSD1 demethylase complex for chromatin association and epithelial-to-mesenchymal transition. J Biol Chem 288: 27680-27691, 2013.

25. Kawata H, Yamada K, Shou Z, Mizutani T, Yazawa T, Yoshino M, Sekiguchi T, Kajitani $\mathrm{T}$ and Miyamoto $\mathrm{K}$ : Zinc-fingers and homeoboxes (ZHX) 2, a novel member of the ZHX family, functions as a transcriptional repressor. Biochem J 373: 747-757, 2003.

26. Lv Z, Zhang M, Bi J, Xu F, Hu S and Wen J: Promoter hypermethylation of a novel gene, ZHX2, in hepatocellular carcinoma. Am J Clin Pathol 125: 740-746, 2006.

27. Nagel S, Schneider B, Meyer C, Kaufmann M, Drexler HG and Macleod RA: Transcriptional deregulation of homeobox gene ZHX2 in Hodgkin lymphoma. Leuk Res 36: 646-655, 2012.

This work is licensed under a Creative Commons Attribution-NonCommercial-NoDerivatives 4.0 International (CC BY-NC-ND 4.0) License. 